\title{
Applying Robust Intelligent Algorithm and Internet of Things to Global Maximum Power Point Tracking of Solar Photovoltaic Systems
}

\author{
En-Chih Chang $\mathbb{D}$ \\ Department of Electrical Engineering, I-Shou University, No. 1, Sec. 1, Syuecheng Rd., Dashu District Kaohsiung City 84001, Taiwan \\ Correspondence should be addressed to En-Chih Chang; enchihchang@isu.edu.tw
}

Received 4 August 2020; Revised 29 October 2020; Accepted 11 November 2020; Published 25 November 2020

Academic Editor: Chao-Yang Lee

Copyright (c) 2020 En-Chih Chang. This is an open access article distributed under the Creative Commons Attribution License, which permits unrestricted use, distribution, and reproduction in any medium, provided the original work is properly cited.

\begin{abstract}
The important dare in the solar photovoltaic (PV) system is to investigate the performance under partial shading conditions. A robust intelligent algorithm (RIA) connected with internet of things (IOT) is developed to offer the real-time monitoring of solar PV systems, thus ensuring global maximum power point tracking (MPPT). The RIA comprises a limited-time terminal sliding-mode control (LTTSMC) and a quantum particle swarm optimization- (QPSO-) radial basis function (RBF) neural network. The LTTSMC creates a quick limited-system-state convergence time and allows for singularity avoidance. However, if the system ambiguity is overrated or underrated, the tremble phenomenon or steady-state error probably occurs around the LTTSMC. The QPSO-RBF neural network is integrated into LTTSMC to handle plant parameter variations and external load perturbations, thus reducing tremble and steady-state errors. With the aggregation of the RIA and the IoT, the remote monitoring in the solar PV system yields faster convergence to nonsingular points, and it also introduces neural network method to achieve more accurate ambiguity estimation. Experimental results show the mathematical analysis and performance enhancement of a prototype algorithm-controlled solar PV system based on digital signal processing under transient and steady-state loading conditions. Because the proposed solar PV system has notable advantages over the classical terminal-sliding solar PV system in terms of tracking accuracy and robust adaptation, this paper is worthy of reference to designers of relative robust control and neural network learning algorithm.
\end{abstract}

\section{Introduction}

The robust intelligent algorithm for the internet of things supports the remote monitoring of real-time solar PV data, such as panel voltage, current, power, temperature, and solar radiation. In order to achieve the maximum power point tracking of a photovoltaic system, the output side of the solar cell needs to be regulated with a switching power converter with maximum power point tracking control, which allows the solar panel to output the maximum power and achieve fast and accurate tracking [1-6]. Various maximum power point tracking (MPPT) methods have been proposed in the literature, such as perturbation observation, incremental conductance, fuzzy control, and linear approximation [710]. However, the solar illumination and ambient temperature are closely relative to the maximum power output of the solar panel. Most of these MPPT algorithms cannot offer a strict convergence and stability analysis or have the problem with fast tracking to the maximum power point, resulting in reduced output power. Sliding-mode control (SMC) is simple and easy to design and has the robustness to parametric variations and external interference during the sliding process [11-17]; many relative SMCs have been published for the control of solar PV systems [18-20]. However, in practice, the stability, convergence, and performance of solar PV systems controlled by the law of the SMC are compromised when the system state convergence time is not finite, and the tremble and steady-state error occurs under the influence of ambiguous interferences. Recently, the limitedtime terminal sliding-mode control (LTTSMC) not only has a robust design method, clear analysis of convergence, and stability, but also provides a limited-system-state conver- 
gence time and improves the dynamic quality of the PV system [21-23]. However, PV arrays are susceptible to partial shading caused by construction, trees, dust, etc. (greatly reduce the output power of the system and result in large energy losses), and therefore the output characteristics of PV arrays may change irregularly, resulting in multiple area extremes of output power. At this moment, using the previously described methodologies of maximum power tracking (such as disturbance observation method, the incremental conductance method, the fuzzy control method, and linear approximation method) would be trapped in the dilemma of tracking to local maximum power points instead of global maximum power points. Some other global optimization algorithms are proposed, such as genetic algorithm (GA), differential evolution (DE), simulated annealing (SA), and ant colony optimization (ACO) [24-27]. But they either converge prematurely to local optimization or search time is too long. Recently, based on the advanced development of artificial intelligence (AI) [28-34], the radial basis function (RBF) neural network can approximate complicated nonlinear functions by using Gaussian kernels and possess fast learning speed, leading to better transient and steady state for MPPT in PV systems. Also, particle swarm optimization (PSO) is a simple and effective method that has been applied to the actual MPPT systems [35-37]. Its improved version QPSO (quantum particle swarm optimization) can address the drawback of regular PSO which converges easily to local solutions, guaranteeing convergence to global optimal solutions [38-41]. Therefore, inspired by good algorithm design concepts and practical applications [42-45], it will be a good idea to combine the QPSO with RBF neural network. Such hybrid learning mechanism uses optimal methodology and neural network into a systematical structure to govern ambiguity, randomness, and time-varying vagueness, endowing with more accurate constraint estimates. For this reason, the LTTSMC with QPSO-RBF neural network can attenuate the tremble when the ambiguity value is overrated or reduce the steady-state error when the ambiguity value is underrated. In other words, by using the QPSO-RBF neural network and system state information, the switching gain of the LTTSMC can be adaptively adjusted to moderate the overconservation of the LTTSMC design, thereby lessening the tremble and offering global maximum power output. When subjected to partial shading and under high-ambiguity conditions, experimental results from digital implementation show that the proposed algorithm will enable the solar maximum power tracking system to enhance the performance during steady state and tracking speed during transient conditions.

\section{Description of Solar PV System}

In order to increase the efficiency of solar PV systems, it is necessary to have a maximum power tracking function. In particular, it should be noted that when the PV array encounters a partially occluded phenomenon, the power-voltage curve shows multiple peaks (Figure 1), and traditional maximum power tracking methods (e.g., perturbation observation, incremental conductance, and hill climbing) will fall into a local maximum power point instead of a global maxi-

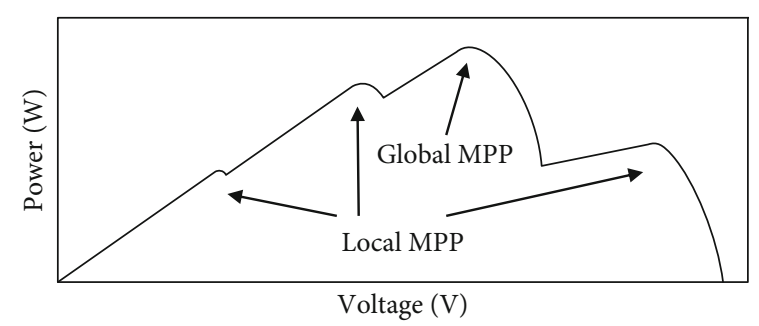

FIgURe 1: Power-voltage curve for partial shading.

mum power point. In addition, there is a voltage reference value corresponding to the maximum power point of a solar PV system. Therefore, a Zeta DC-DC converter (Figure 2) is used to adjust the solar cell voltage (for the maximum power point voltage). This is because the output voltage of Zeta DCDC converter can be higher/lower than the input voltage and have the same polarity with continuous output current. Due to the small-ripple inductor current, it can reduce the tremble during steady-state sliding mode.

For solar PV systems, we propose a QPSO-RBF neural network which calculates a voltage reference at the maximum power point in a solar array under partial shading conditions, and a limited-time terminal sliding-mode control achieves the $v_{\mathrm{PV}}$ to reach the voltage reference. The system state variables of the solar PV system are derived from the following matrix. The solar Zeta DC-DC converter circuit shown in Figure 2 uses the switch on and off states to express the matrix of the solar PV system as follows:

$$
\dot{x}(t)=f(x(t))+g(x(t)) u
$$

where $\dot{x}=\left[\begin{array}{lllll}\dot{v}_{\mathrm{PV}} & \dot{i}_{L 1} & \dot{i}_{L 2} & \dot{v}_{c 1} & \dot{v}_{c 2}\end{array}\right]^{T}, u$ denotes control input with duty cycle signal, $f=\left[\begin{array}{lllll}i_{\mathrm{PV}} / C_{i} & -v_{c 1} / L_{1} & -v_{c 2} / L_{2} & i_{L 1} / C_{1} & \left(i_{L 2}-i_{o}\right) /\end{array}\right.$ $\left.C_{o}\right]^{T}$, and $g=\left[\begin{array}{lll}-\left(i_{L 1}+i_{L 2}\right) / C_{i} & v_{\mathrm{PV}}+v_{c 1} & \left(v_{\mathrm{PV}}+v_{c 1}\right) / L_{2}\end{array}-\left(i_{L 2}\right.\right.$ $\left.\left.+i_{L 1}\right) / C_{1} \quad 0\right]^{T}$. It should also be noted that the parameters of the Zeta DC-DC converter need to be appropriately designed as suggested below [46-49]. In actual coupled inductor, the inductors have unequal inductance, and the ripple currents are not completely the same. For the required ripple current value, the inductance of the coupled inductor can be estimated to be half of the required inductance when two independent inductors $\left(L_{1}\right.$ and $\left.L_{2}\right)$ exist; this can be referred to (4) and (19) in [46, 47], respectively. The coupling capacitor $C_{1}$ shown as (6) in [48] can be designed based on the ripple voltage. Also, (19) in [49] shows that the output capacitor $C_{o}$ is recommended to have sufficient capacitance to keep the DC-link voltage and to provide continuous load current under high switching frequency.

The control objective in a PV system is to make $v_{\mathrm{PV}}$ track the reference voltage $v_{r}$. The error $e_{1}=v_{\mathrm{PV}}-v_{r}$ can be defined as the difference of the $v_{\mathrm{PV}}$ and the reference voltage, at which the control rule is designed, and the purpose is to design the control rule well. Then, to convert the DC voltage generated from Zeta DC-DC converter to AC voltage, Figure 3 shows the circuit diagram of a single-phase fullbridge voltage source inverter, followed by LC filter. The full-bridge DC-AC inverter is the core of the system, which 


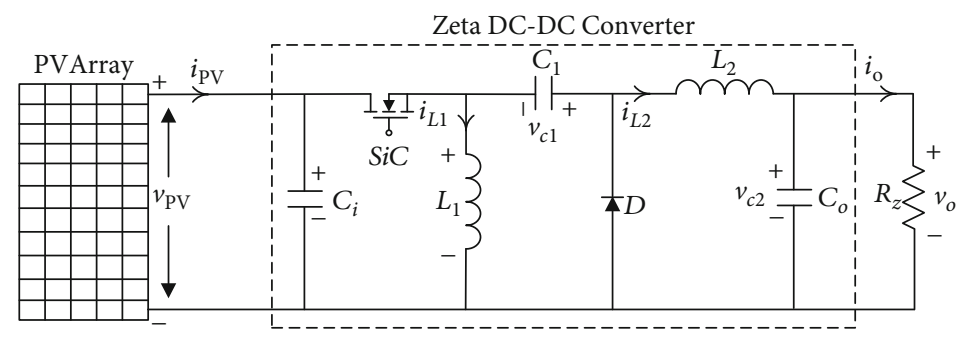

FIgURE 2: Solar PV powered Zeta DC-DC converter.

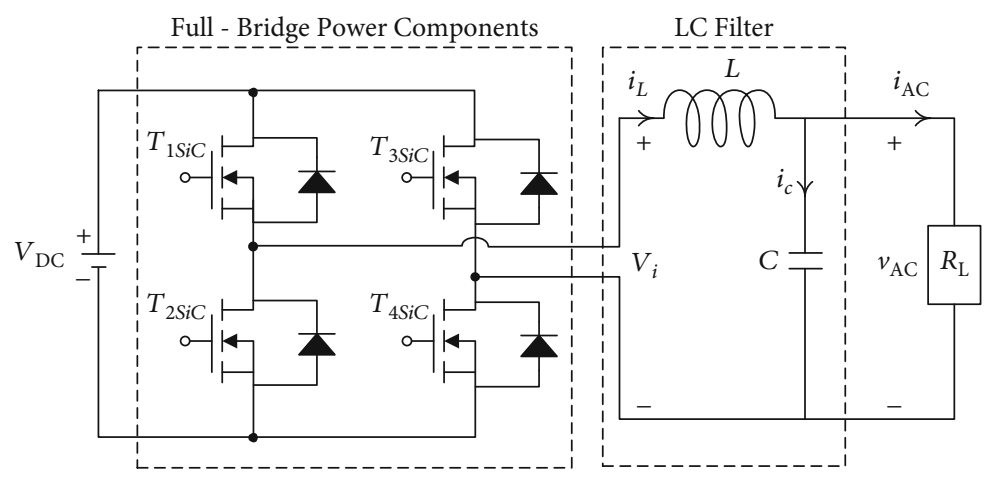

FIGURE 3: Block diagram of single-phase DC-AC inverter.

converts the DC input chopper into a series of pulse-width modulation according to the modulation signal. The function of second-order LC filter is to eliminate the high-frequency component of chopper output voltage $v_{i}$. We also need to pay attention to the selection and determination of the LC filter component values of the full-bridge DC-AC inverter, which can refer to the recommendations in the following literature [50-52]. (1) Selection of switching frequency [51, 52]: usually in order to reduce the size of the LC filter, the switching frequency of the insulated gate bipolar transistor switches can be picked from $3 \mathrm{kHz}$ to $15 \mathrm{kHz}$, and the silicon carbide MOSFET switches can be chosen higher than $30 \mathrm{kHz}$ switching frequency. (2) Selection of a factor value relative to LC low-pass filter cutoff frequency [50]: when there is a greater factor value, the switching and fundamental frequencies yield more descent and less amplification, respectively. Once the suggested modulation value is lower than 0.95 , the least value of this factor can be obtained. (3) Selection of a factor value relative to the switching frequency and the inductor ripple current [52]: the inductor ripple current limit value of $20 \%$ to $40 \%$ can be recommended. The factor is chosen by the equations (8), (20), (25), and (26) of [50], thereby acquiring the $L$ and $C$ values. Applying KVL and KCL, the state space equation for a single-phase inverter can be expressed as $\ddot{v}_{A C}=-v_{A C} / L C-\dot{v}_{A C} / R_{L} C+k_{p w m} \cdot u_{\text {inv }} / L C$, where $k_{p w m}$ stands for the equivalent gain of the inverter. Therefore, the error state equation of the inverter is obtained as $\dot{x}_{e 2}=-x_{e 1} / L$ $C-x_{e 2} / R_{L} C+k_{p w m} \cdot u_{i n v} / L C-v_{A C, r} / L C-\dot{v}_{A C, r} / R_{L} C-\ddot{v}_{A C, r}$, where $x_{e 1}=v_{\mathrm{AC}}-v_{\mathrm{AC}, r}, \dot{x}_{e 1}=x_{e 2}=\dot{v}_{\mathrm{AC}}-\dot{v}_{\mathrm{AC}, r}$, and $v_{\mathrm{AC}, r}$ is a demanded sinusoidal reference. Figure 4 displays the structure of the whole control system, and in order to allow the error states converged to zero, the control law $u_{\text {inv }}$ is designed via fractional proportional-integral (FPI) method expressed as $x_{e 1} \cdot\left(k_{p}+k_{i}\left(d^{-\alpha} / d t^{-\alpha}\right)\right)$, where $k_{p}$ is proportional gain, $k_{i}$ denotes integral gain, and $\alpha$ symbols the noninteger order of the integral term. It must be mentioned that the FOMCON toolbox of the Matlab/simulink package software offers FPI control applications, such as the identification of dynamic models in the time domain and frequency domain, and controller design. This paper employs the FOMCON toolbox to get the gain values of $k_{p}$ and $k_{i}$, and then these gain values are adopted in digital implementation, thereby achieving good inverter response [53-56].

\section{Control Design}

The limited-time sliding function can be written as

$$
s=e_{1}+\frac{1}{\lambda} e_{2}^{q / p}
$$

where $e_{1}=v_{\mathrm{PV}}-v_{r}, e_{2}=\dot{e}_{1}, \lambda>0$, and $p$ and $q$ intend by positive odd numbers $(p<q<2 p)$. Afterward a sliding-mode reaching rule $\dot{s}=-\eta_{1}|s|^{1-\gamma_{1}} \operatorname{sgm}(s)-\eta_{2}|s|^{\gamma_{2}} \operatorname{sgm}(s)$ is recommended to be employed. Note that a sigmoid function sgm( $s)=s / \tau / \sqrt{1+(s / \tau)^{2}}$; here, $\tau$ is a small positive constant. The control law of the LTTSMC can be expressed as

$$
u=u_{\mathrm{eq}}+u_{s}
$$




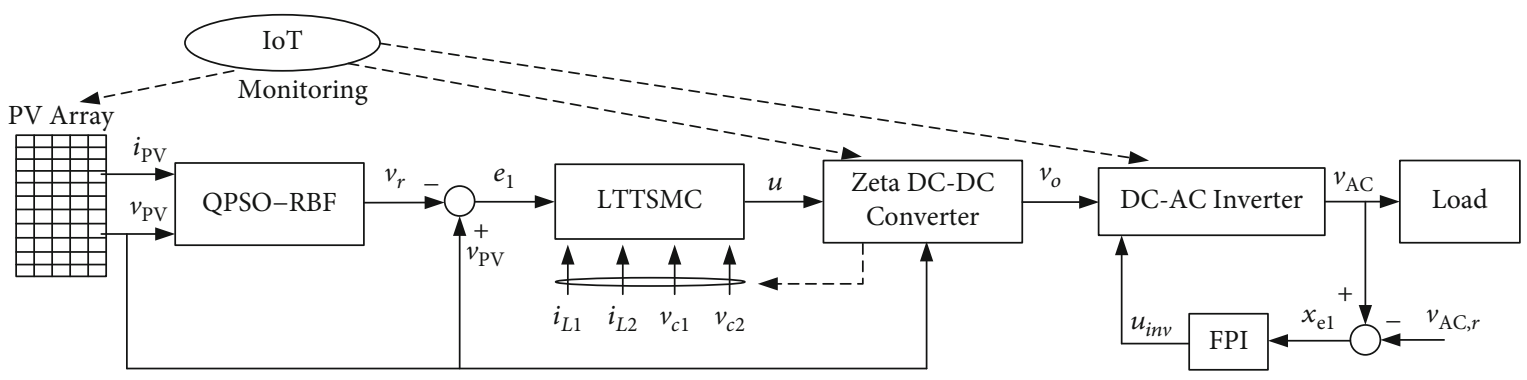

FIGURE 4: Block diagram of total control system.

Since $u_{\text {eq }}$ is the equivalent control without interferences, it can be written succinctly as

$$
u_{\mathrm{eq}}=-g^{-1}\left(k_{\mathrm{eq}} e+\lambda \frac{p}{q} e_{2}^{2-q / p}\right)
$$

where $e$ is the error state vector and $k_{\mathrm{eq}}$ stands for equivalent feedback gain. The index of the $e_{2}$ in the equivalent control $u_{\text {eq }}$ is greater than zero, leading to nonsingularity.

Also,

$u_{s}=-g^{-1}\left[\eta_{1}|s|^{1-\gamma_{1}} \operatorname{sgm}(s)+\eta_{2}|s|^{\gamma_{2}} \operatorname{sgm}(s)\right], \eta_{1}, \eta_{2}>0,0<\gamma_{1}<1, \gamma_{2}>1$,

where the sliding control $u_{s}$ can compensate for the repercussions of agitations. As a result, the system state will be forced to arrive $s=0$ and converge in a limited amount of time.

Proof. Specify a Lyapunov candidate as

$$
V=\frac{1}{2} s^{2}
$$

According to the dynamic system trajectory and the control law (3) and utilizing the above Lyapunov candidate, the time derivative $V$ is given as

$$
\dot{V}=s \dot{s}=s \cdot\left(\dot{e}_{1}+\frac{1}{\lambda} \frac{q}{p} e_{2}^{q / p-1} \dot{e}_{2}\right) \leq-s \cdot\left(\frac{1}{\lambda} \frac{q}{p} e_{2}^{q / p-1}\left(\eta_{1}|s|^{1-\gamma_{1}}+\eta_{2}|s|^{\gamma_{2}}\right) \operatorname{sgm}(s)\right) .
$$

On account of $e_{2}^{q / p-1}>0$, the differential of $V$ is less than or equal to zero. This deduced that the $s$ and $\dot{s}$ of the LTTSMC in (7) are allowed to converge to the equilibrium for a limited time. In the case of $s=e_{1}+\lambda^{-1} e_{2}^{q / p}$ and $\dot{s}=-\eta_{1}$ $|s|^{1-\gamma_{1}} \operatorname{sgm}(s)-\eta_{2}|s|^{\gamma_{2}} \operatorname{sgm}(s)$, the system state (1) must also quickly converge to the equilibrium within a limited time. However, the tremble or steady-state error emerges from the LTTSMC. The reason for this situation is that the system load has drastic changes or extreme nonlinearity, so that the final system output cannot follow the reference sine waveform, resulting in inaccurate tracking performance. The control signal $u$ derived from (3) is altered by the addendum of the QPSO-RBF neural network method, which depresses the tremble/steady-state error in the solar PV system. The

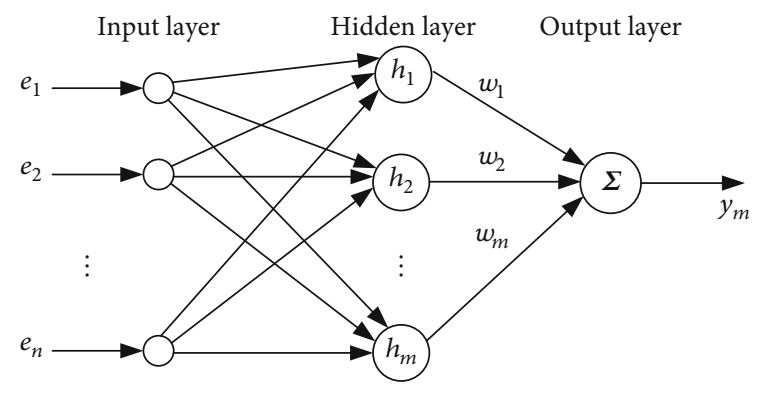

FIGURE 5: Constitution of RBF neural network.

RBF neural network is depicted as Figure 5. It is easy to grasp that there are three layers in the RBF neural network constitution, namely, the input layer, the hidden layer, and the output layer. The input layer only carries out the delivery of the information, the hidden layer possesses a perceptible region containing a set of RBFs, and the output layer neurons represent linear amalgamations of neuron activations in the hidden layer. More concretely, there are nonlinear interconnections amid the input layer and the hidden layer, but there are linear relationships amid the hidden and the output layers. Using these relationships, a mapping of lowdimensional vectors is performed, and the RBF constitution transforms the low-dimensional linearly inseparable space into a high-dimensional linearly divisible space, thereby providing an approximation ability of the uncertain nonlinear functions. In general, it is not effortless to precisely calculate the upper limit of a system subject to parameter uncertainty and external intermission, but the RBF constitution is a useful tool for solving highly complex and nonlinear problems. Thereby, this RBF neural network is used to estimate the upper limit of system uncertainty and external intermission, effectively depressing the effect of the tremble. With the application of solar PV systems, since the neurons use mathematical operations in the hidden layer and output layers, the tracking error vector $e$ is given as input data in the input layer, and thereafter the processed input is passed through the Gaussian RBF, which maps the processed input to the output layer to obtain the output function. Note that the most effective way to discover a particular data set is to use systematic experimentation. It is often not easy to analytically calculate the number of layers or nodes used in each layer of an artificial neural network to solve the specific problem of actual prediction modeling. This is due to the fact that the number of layers and nodes in each layer must be specified 
as model hyperparameters. The designer may be the first person to try to solve the own particular problem using neural networks. As a result, we must use powerful testing tools and controlled experiments to discover the answer. The $\mathrm{R}$ programming language provides a package called caret that helps determine the numbers of layers and nodes used in RBF neural network to achieve the minimum mean square error (MSE), i.e., the best combination of parameters [5759]. Thereby, this constitution is used to adjust the LTTSMC gain to depress tremble and improve the performance of the system, as described and demonstrated below.

We first define the upper limit of the plant parameter changes and the external load intermissions as $\bar{\Theta}>|\Theta|$, and the estimated value is symbolized by $\widetilde{\bar{\Theta}}=W^{T} h\left(e_{i}\right)$; here, $W=\left[w_{1}, w_{2}, \cdots, w_{m}\right]^{T}$ stands for network weights, and $h$ denotes the Gaussian function as follows:

$$
h_{j}=\exp \left(-\left\|e-c_{j}\right\|^{2} / 2 \rho_{j}^{2}\right), j=1,2, \cdots, m
$$

where $c_{j}$ represents the center of the hidden layer neuron, and the smoothing parameter $\rho_{j}$ means the width of Gaussian function. Then, make the following suppositions throughout the proof.

Supposition $I[60,61]$ : if there is an optimal weight matrix $W^{*}$ of the RBF constitution and the (9) is hold, then the output of the optimal neural network can be obtained.

$$
\delta\left(e_{i}\right)=W^{* T} h\left(e_{i}\right)-\bar{\Theta}<\delta_{n} .
$$

Supposition II [60, 61]: presuming the uncertainty upper limit of system (1) satisfies the following form:

$$
\bar{\Theta}-|\Theta|>\delta_{0}>\delta_{n}>0
$$

Then, the sliding control $u_{s}$ can be redescribed as

$$
u_{s}=-\widetilde{\bar{\Theta}} \operatorname{sgn}(s) \text {. }
$$

The stability of the system (1) can be guaranteed by Lyapunov function below.

$$
V=\frac{1}{2} \cdot\left(s^{2}+\kappa^{-1}\left(W^{*}-W\right)^{T}\left(W^{*}-W\right)\right)
$$

where $\kappa>0$.

Using the above-mentioned equivalent control and (11), the derivative of $V$ yields

$$
\begin{aligned}
\dot{V} & =s \dot{s}-\kappa^{-1}\left(W^{*}-W\right)^{T} \dot{W} \\
& =-|s| \widetilde{\widetilde{\Theta}}+s \Theta-\kappa^{-1}\left(W^{* T}-W^{T}\right) \dot{W} .
\end{aligned}
$$

In addition, the weight update method is designed to

$$
\dot{W}=\kappa|s| h\left(e_{i}\right) .
$$

TABLE 1: Solar cell electrical parameters.

\begin{tabular}{lc}
\hline (Irradiances $1 \mathrm{~kW} / \mathrm{m}^{2}$, module temperature $\left.25^{\circ} \mathrm{C}\right)$ & $75 \mathrm{~W}$ \\
Rated power & $17 \mathrm{~V}$ \\
\hline Rated working voltage & $4.4 \mathrm{~A}$ \\
Rated working current & $21.7 \mathrm{~V}$ \\
Open-circuit voltage & $4.8 \mathrm{~A}$ \\
Short-circuit current & 1.2 \\
Ideality factor of the diode & $4 \mathrm{~mA} /{ }^{\circ} \mathrm{C}$ \\
Temperature coefficient of short-circuit current & 2.035 \\
P-N junction parameter &
\end{tabular}

From (9), (10), and (14), the (13) becomes

$$
\dot{V}=-|s| W^{* T} h\left(e_{i}\right)+s \Theta \leq-|s|\left(\bar{\Theta}+\delta\left(e_{i}\right)-|\Theta|\right) \leq-|s|\left(\delta_{0}-\delta_{n}\right) \leq 0,
$$

where $\kappa=\delta_{0}-\delta_{n}$. Although the (15) is kept, it is necessary to give further basic explanations about the presence of compact sets in the control of neural networks so that the approximation capability of the neural networks can be constructed. In order to limit the state of the closed-loop system, the reports of the necessary assumptions, the use of a backstepping methodology, and the suggestion for an adaptive law have been provided and proved from previous work, leading to a semiglobally consistent ultimate bounded system stabilization that always keeps the system state in a compact set (see [62] and references therein). Eventually, the solar PV system with RBF neural network and LTTSMC becomes progressively stable and then achieves finite-time convergence with zero tracking error. However, the traditional RBF neural network adopts the local information based on the parameter space to set the relative parameters, which results in the values of the parameters $c_{j}, \rho_{j}$, and $w_{j}$ becoming locally optimal solutions. To obtain the global optimal solution, the QPSO algorithm can be used. When the number of overlaps approaches infinity, there exists a traditional PSO whose algorithm cannot converge to the global optimum with probability 1, i.e., it is not globally convergent. In addition, the velocity of a single particle has an upper limit on its search space, which is finite and cannot cover the entire feasible solution space, thus limiting the algorithm's ability to search the entire domain. Instead of requiring velocity vectors, the QPSO is a completely stochastic stacking equation that has no deterministic trajectory as the particle moves through the quantum space, and the entire stacking equation has fewer easily controllable parameters, allowing the particle to search for the global optimal value over the entire feasible solution space. The superposition representation of the QPSO is expressed as

$$
\zeta_{i}^{k}=\psi^{k} \zeta_{p \text { best }, i}^{k}+\left(1-\psi^{k}\right) \zeta_{g \text { best }, i}^{k}
$$

$$
X_{i}^{k+1}=\zeta_{i}^{k} \pm \beta^{k}\left|Q^{k}-X_{i}^{k}\right| \ln \left(\frac{1}{\Psi^{k}}\right)
$$




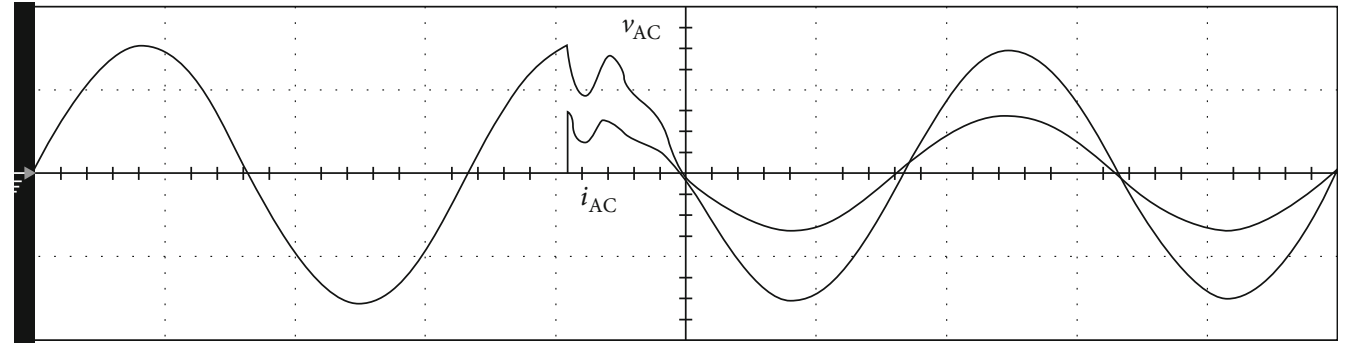

FIGURE 6: Experimental output waveforms of a solar PV system controlled by the classical terminal sliding-mode control under step load (vertical: $100 \mathrm{~V} / \mathrm{div}$ and $5 \mathrm{~A} / \mathrm{div}$; horizontal: $5 \mathrm{~ms} / \mathrm{div}$ ).

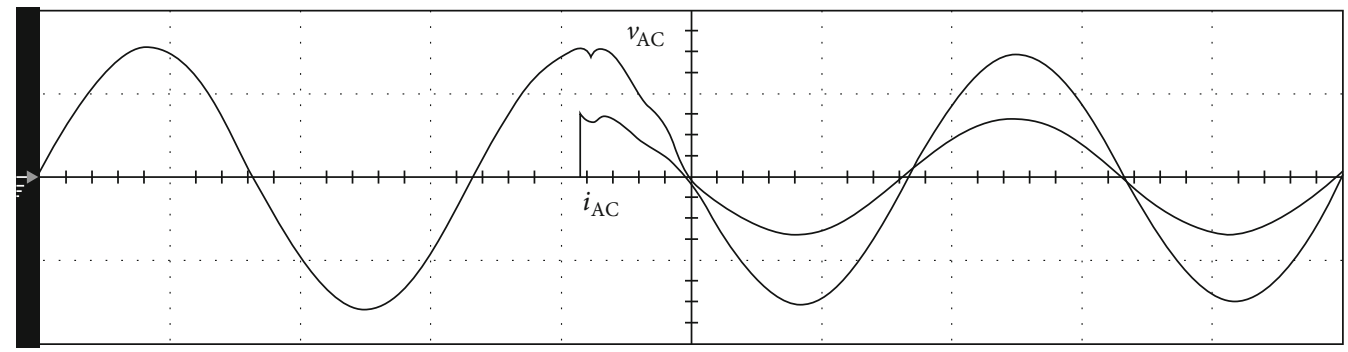

FIGURE 7: Experimental output waveforms of a solar PV system controlled by the proposed algorithm under step load (vertical: $100 \mathrm{~V} /$ div and $5 \mathrm{~A} /$ div; horizontal: $5 \mathrm{~ms} / \mathrm{div})$.

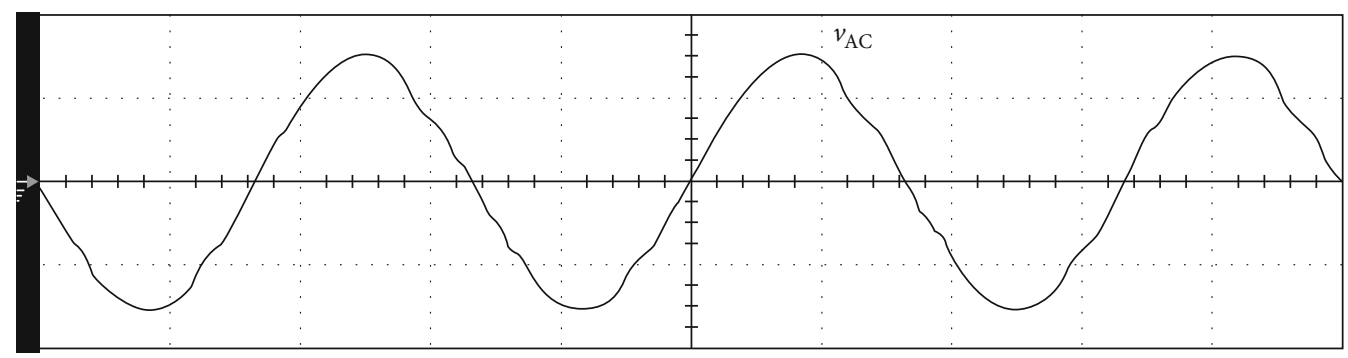

FIGURE 8: Experimental output waveforms of a solar PV system controlled by the classical terminal sliding-mode control under the variations of filter parameters (vertical: $100 \mathrm{~V} / \mathrm{div}$; horizontal: $5 \mathrm{~ms} / \mathrm{div}$ ).

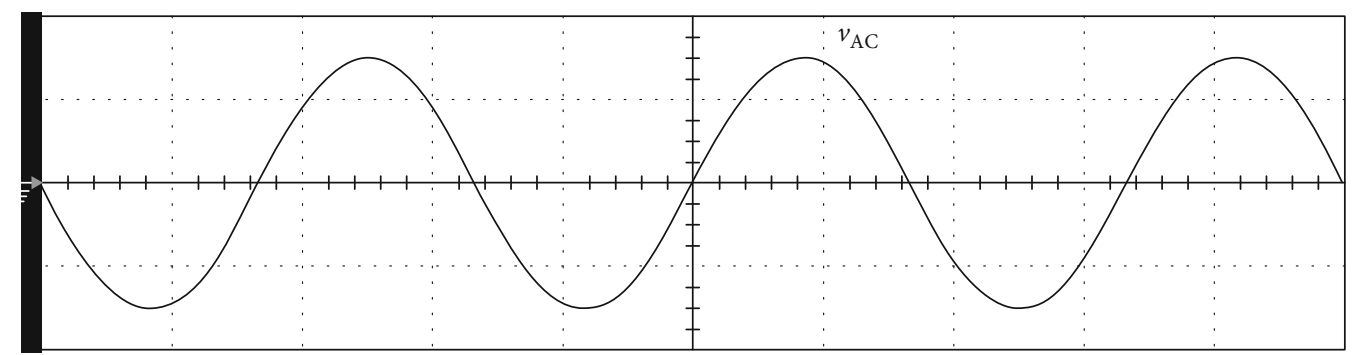

FIGURE 9: Experimental output waveforms of a solar PV system controlled by the proposed algorithm under the variations of filter parameters (vertical: $100 \mathrm{~V} /$ div; horizontal: $5 \mathrm{~ms} / \mathrm{div}$ ).

where $\beta^{k}=\beta_{\text {base }}+\Re$ indicates the compression/expansion factor; here, $\beta_{\text {base }}$ implies the basic compression/expansion factor, and $\mathfrak{R}$ represents a random number evenly distributed over $(0,1) ; \psi^{k}$ and $\Psi^{k}$ represent random numbers including uniform distribution between 0 and 1 , and $Q^{k}$ denotes the average of the best locations in the region of the cluster for all particles in the search history.

\section{Results and Discussion}

In order to verify the effectiveness of the proposed controller, the solar module with 4 series and 2 parallel is used, and Table 1 gives relevant parameters. Based on the design of Zeta converter and considering the actual PV environment, the parameters are provided as follows: the inductance $L_{1}$ is $0.46 \mathrm{mH}$, the inductance $L_{2}$ denotes $0.5 \mathrm{mH}$, the capacitance 

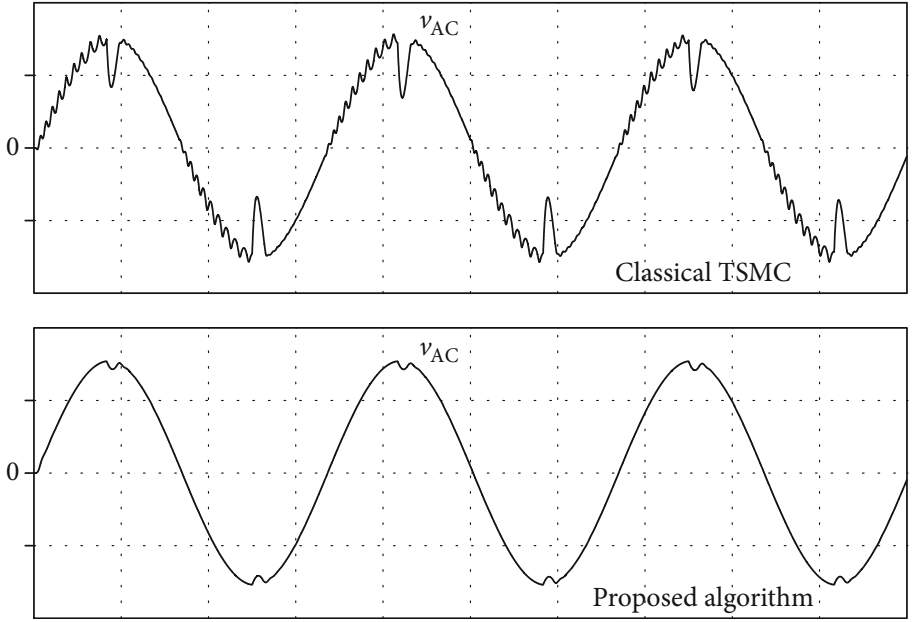

FIGURE 10: Simulated performance of a solar PV system controlled by the classical TSMC and the proposed algorithm under partial shading condition with five local peaks (vertical: $100 \mathrm{~V} /$ div; horizontal: $5 \mathrm{~ms} / \mathrm{div}$ ).

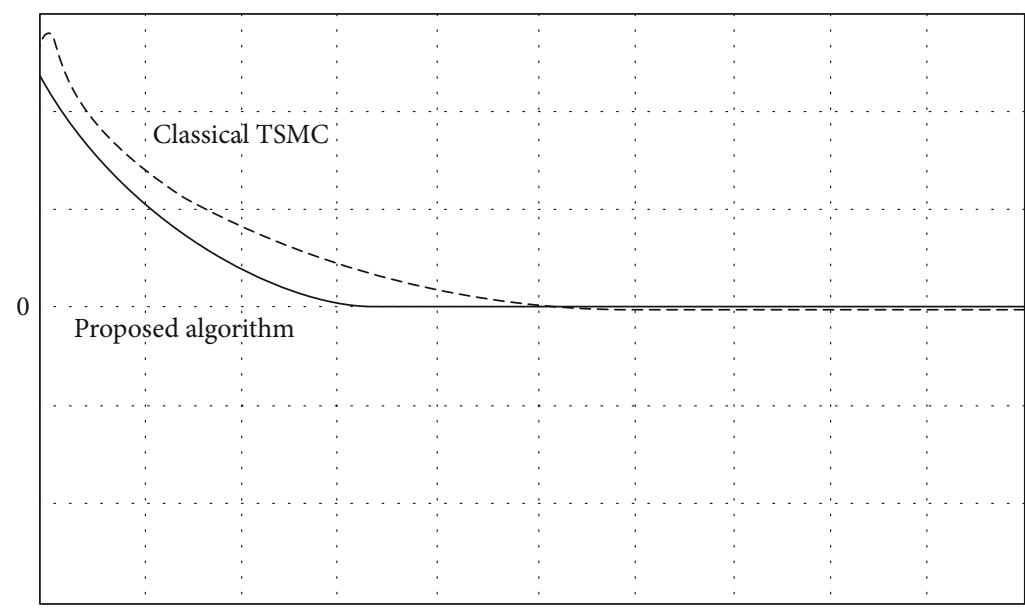

FIGURE 11: Tracking error comparison between the classical TSMC and the proposed algorithm (vertical: 0.15 V/div; horizontal: 0.75 $\mathrm{ms} / \mathrm{div})$.

$C_{1}$ indicates $100 \mu \mathrm{F}$, the capacitance $C_{i}$ is $120 \mu \mathrm{F}$, and the capacitance $C_{o}$ means $1000 \mu \mathrm{F}$. Also, the inductance $L$ of the DC-AC inverter stands for $0.4 \mathrm{mH}$, the capacitance $C$ is $10 \mu \mathrm{F}$, the DC-link voltage $V_{\mathrm{DC}}$ signifies $200 \mathrm{~V}$, the AC output is $110 \mathrm{Vrms}, f=60 \mathrm{~Hz}$, and the switching frequency represents $30 \mathrm{kHz}$.

Figure 6 shows the experimental output waveform of a classical PV system under partial shielding at a 90 trigger angle, changing from no load to full load. It can be seen from the figure that the transient voltage drop does not regain quickly, and the ability of the controller to compensate for the transient voltage is apparently poor. Figure 7 reports the experimental output waveform when the PV system encounters partial shielding using the proposed algorithm at 90 trigger angle, changing from no-load to full-load condition; we can observe that the transient voltage drop regains within a short period of time, and the compensation ability is better than the classical terminal sliding-mode control. Figure 8 illustrates the experimental output voltage of the PV system under partial shielding condition with classical terminal sliding-mode control when the filter parameters of the PV system are changed. The proposed output voltage of a PV system under partial shielding has depicted in Figure 9 shows when the filter parameters of the PV system are changed. Obviously, the output voltage waveform of the PV system controlled by the classical terminal sliding-mode control has more distortion, but the proposed output voltage of the PV system is very close to the sinusoidal reference voltage waveform. Considering the TRIAC-controlled load situation, the simulated output voltage waveform of a solar PV system controlled by the proposed algorithm is displayed in Figure 10 under partial shading condition with five local peaks. The proposed algorithm implies the strong performance with a slight voltage sag and rapid retrieval time. Figure 11 depicts the tracking error comparison between the classical TSMC and the proposed algorithm. The tracking accuracy and speed of the proposed algorithm are significantly better than classical TSMC. Table 2 shows the output voltage drop in step loads, and Table 3 displays the variation of the output voltage THD on the filter parameters. It is 
TABLe 2: Output voltage drop under step load.

\begin{tabular}{lcc}
\hline & Classical TSMC & Proposed algorithm \\
\hline Step loads & No load to full load & No load to full load \\
Voltage drop & $60.2 \mathrm{Vmax}$ & $10.2 \mathrm{Vmax}$ \\
Improvement degree & $\begin{array}{c}\text { Nearly eighty-four } \\
\text { in voltage drop reduction }\end{array}$ \\
\hline
\end{tabular}

TABLE 3: Output voltage \%THD under the variations of filter parameters.

\begin{tabular}{lcc}
\hline & Classical TSMC & Proposed algorithm \\
\hline Filter parameters & $10 \% \sim 200 \%$ & $10 \% \sim 200 \%$ \\
$\%$ THD & $20.6 \%$ & $0.015 \%$ \\
Improvement degree & Complete improvement the impact \\
& when faced with filter parameter changes \\
\hline
\end{tabular}

important to point out that we can compare and analyze four kinds of lately used optimization algorithms, such as adaptive differential evolution (ADE), enhanced simulated annealing (ESA), improved genetic algorithm (IGA), and discrete particle swarm optimization (DPSO) [63-66]. The generation mechanism of ADE new solutions and the population search capability are similar to the DPSO. Because the best solution in the population has no impact on other solutions, there is better diversity but the mutation vector usually comes from the nonoriginal of solutions. The DPSO has fewer iterations, and its performance is much better than ESA. A lot of iterations are demanded by SA, so as to get the DPSO identical results; nevertheless, each iteration time of DPSO wastes more time than ESA. With the probability, both parents and individuals can be selected. The crossover operation can produce seed, which extracts from the parent, and its solution is similar to the parent. The IGA tends to generate solutions that can be clustered around certain fine solutions in the aggregate. By introducing different mutation operations, the diversity of IGA can be introduced into the solution, but the increment in the solution time appears nonlinearity while the population increases.

\section{Conclusions}

The proposed algorithm uses a QPSO-RBF neural network to find the global maximum power point of the photovoltaic array in the event of partial shielding and a limited-time terminal sliding-mode control to provide tracking control with the unique advantage of finite-time system state convergence, depressed tremble, and steady-state error under uncertain intermission conditions. The algorithm developed with the help of the IoT is used in the solar PV system to establish the maximum power output of solar panels and to maintain the highest PV energy conversion efficiency during partially shaded conditions. When comparing the output voltages of the classical terminal sliding-mode control under varying filter parameters, the total harmonic distortion (THD) rate of the proposed control is very low, and the voltage waveform is close to the required sinusoidal reference voltage. Thereby, the proposed experiments in the solar system indeed achieve good performance under partial shading conditions. More importantly, the IoT depresses the tedious task of visiting working sites with frequently recording of performance data, therefore enhancing the control of remote areas for effective and speedy troubleshooting and maintenance.

\section{Data Availability}

The data used to support the findings of this study are included within the article.

\section{Conflicts of Interest}

The author declares that there is no conflict of interest regarding the publication of this article.

\section{Acknowledgments}

The author gratefully acknowledges the financial support of the Ministry of Science and Technology, Taiwan, R.O.C., under project numbers MOST 109-3116-F-006-020-CC1 and MOST 107-2221-E-214-006. The author also gratefully acknowledges the financial support of the 2020 ISU Research Project, under contract number ISU-109-01-01A.

\section{References}

[1] P. Mohanty, T. Muneer, K. Tariq, and L. Mohan, Solar Photovoltaic System Applications, Springer, New York, 2015.

[2] B. M. Wilamowski and J. D. Irwin, Power Electronics and Motor Drives, CRC Press, Boca Raton, FL, USA, 2011.

[3] M. S. Mahmoud, Microgrid: Advanced Control Methods and Renewable Energy System Integration, Elsevier Science Ltd., Amsterdam, The Netherlands, 2016.

[4] F. Blaabjerg, Control of Power Electronic Converters and Systems, Academic Press, Cambridge, MA, USA, 2018.

[5] R. Yuriy, R. Sergey, C. Evgeny, and V. Pavel, Power Electronics Basics: Operating Principles, Design, Formulas, and Applications, CRC Press, Boca Raton, FL, USA, 2016.

[6] L. D. Branko and B. Branko, Power Electronics: Converters and Regulators, Springer, New York, 2015.

[7] M. Gangavarapu, "Perturb and observe MPPT algorithm implementation for PV applications," International Journal of Computer Science and Information Technologies, vol. 6, no. 2, pp. 1884-1887, 2015.

[8] R. I. Putri, S. Wibowo, and M. Rifa'i, "Maximum power point tracking for photovoltaic using incremental conductance method," Energy Procedia, vol. 68, pp. 22-30, 2015.

[9] B. Bendib, F. Krim, H. Belmili, M. F. Almi, and S. Boulouma, "Advanced fuzzy MPPT controller for a stand-alone PV system," Energy Procedia, vol. 50, pp. 383-392, 2014.

[10] Y. Y. Yang, W. D. Yi, and K. W. Jwo, "High efficiency MPPT using piecewise linear approximation and temperature compensation," Advanced Materials Research, vol. 772, pp. 658663, 2013.

[11] G. Bartolini, L. Fridman, A. Pisano, and E. Usai, Modern Sliding Mode Control Theory, Springer-Verlag, Berlin, 2008.

[12] A. T. Azar and Q. M. Zhu, Advances and Applications in Sliding Mode Control Systems, Springer, New York, 2015. 
[13] H. Sira-Ramirez, "Sliding regimes in general non-linear systems: a relative degree approach," International Journal of Control, vol. 50, no. 4, pp. 1487-1506, 1989.

[14] Z. Doulgeri, "Sliding regime of a nonlinear robust controller for robot manipulators," IEE Proceedings-Control Theory and Applications, vol. 146, no. 6, pp. 493-498, 1999.

[15] S. C. Tan, Y. M. Lai, and C. K. Tse, Sliding Mode Control of Switching Power Converters: Techniques and Implementation, CRC Press, Boca Raton, FL, USA, 2012.

[16] V. Utkin, "Variable structure systems with sliding modes," IEEE Transactions on Automatic Control, vol. 22, no. 2, pp. 212-222, 1977.

[17] H. B. Zhou, J. H. Song, and S. M. Song, "Sliding Mode Guidance Law Considering Missile Dynamic Characteristics and Impact Angle Constraints," International Journal of Automation and Computing, vol. 15, no. 2, pp. 218-227, 2018.

[18] L. G. Wu, P. Shi, and X. J. Su, Sliding Mode Control of Uncertain Parameter-Switching Hybrid Systems, Wiley, New York, 2014.

[19] Z. Wang, Y. Mao, Z. Hu, and Y. Xie, "A sliding mode control design based on the reaching law for matrix rectifiers," Journal of Power Electronics, vol. 16, no. 3, pp. 1122-1130, 2016.

[20] S. Vaidyanathan and C. H. Lien, Applications of Sliding Mode Control in Science and Engineering, Springer, New York, 2017.

[21] Y. X. Zhao, T. Wu, and Y. Ma, "A double power reaching law of sliding mode control based on neural network," Mathematical Problems in Engineering, vol. 2013, 9 pages, 2013.

[22] H. Wang, X. Zhao, and Y. Tian, "Trajectory tracking control of $\mathrm{XY}$ table using sliding mode adaptive control based on fast double power reaching law," Asian Journal of Control, vol. 18, no. 6, pp. 2263-2271, 2016.

[23] C. Y. Dong, H. J. Wang, and W. Y. Cui, "Application of the sliding mode control approach based on double power exponential reaching law for the hydraulic servo system," Applied Mechanics and Materials, vol. 741, pp. 655-658, 2015.

[24] Z. Wang, M. Chinthavali, S. L. Campbell, T. Wu, and B. Ozpineci, "A 50-kW air-cooled SiC inverter with 3-D printing enabled power module packaging structure and genetic algorithm optimized heatsinks," IEEE Transactions on Industry Applications, vol. 55, no. 6, pp. 6256-6265, 2019.

[25] M. Ali, A. Iqbal, M. A. Anees, M. R. Khan, K. Rahman, and M. Ayyub, "Differential evolution-based pulse-width modulation technique for multiphase MC," IET Power Electronics, vol. 12, no. 9, pp. 2224-2235, 2019.

[26] J. P. F. Trovão, V. D. N. Santos, P. G. Pereirinha, H. M. Jorge, and C. H. Antunes, "A simulated annealing approach for optimal power source management in a small EV," IEEE Transactions on Sustainable Energy, vol. 4, no. 4, pp. 867-876, 2013.

[27] S. Yang, M. Wu, X. Yao, and J. Jiang, "Load modeling and identification based on ant colony algorithms for EV charging stations," IEEE Transactions on Power Systems, vol. 30, no. 4, pp. 1997-2003, 2015.

[28] Q. C. Duan, M. X. Mao, P. Duan, and B. Hu, “Application of improved radial basis function neural network method in global MPPT for PV array," in 2015 IEEE Energy Conversion Congress and Exposition (ECCE), pp. 3260-3264, Montreal, QC, Canada, 2015.

[29] H. Hichem, B. R. Chiheb, and Z. F. Abderrahmen, "Performance improvement of a photovoltaic system with a radial basis function network based on particle swarms optimization," in Proc. Int. Conf. Signal, Control and Communication (SCC), pp. 156-162, Hammamet, Tunisia, Tunisia, 2019.
[30] B. N. Roodsari, C. J. B. Macnab, and E. P. Nowicki, "A novel adaptive controller using radial basis function neural network for the wind energy conversion system," in Proc. IEEE Int. Conf. Industrial Technology (ICIT), pp. 715-720, Toronto, ON, Canada, 2017.

[31] E. Kass, T. Eden, and N. Brown, Analysis of Neural Data, Springer-Verlag, New York, 2014.

[32] L. Chen, C. Liu, R. Wu, Y. He, and Y. Chai, "Finite-time stability criteria for a class of fractional-order neural networks with delay," Neural Computing and Applications, vol. 27, no. 3, pp. 549-556, 2016.

[33] J. S. R. Jang, C. T. Sun, and E. Mizutani, Neuro-Fuzzy and Soft Computing: A Computational Approach to Learning and Machine Intelligence, Pearson Education, New Delhi, India, 2015.

[34] K. L. Du, Neural Networks in a Softcomputing Framework, Springer-Verlag, London, 2006.

[35] K. Ishaque and Z. Salam, "A Deterministic Particle Swarm Optimization Maximum Power Point Tracker for Photovoltaic System under Partial Shading Condition," IEEE Transactions on Industrial Electronics, vol. 60, no. 8, pp. 3195-3206, 2013.

[36] K. E. Parsopoulos and M. N. Vrahatis, Particle Swarm Optimization and Intelligence: Advances and Applications, Information Science Reference, Hershey, PA, USA, 2010.

[37] R. Ruiz-Cruz, E. N. Sanchez, F. Ornelas-Tellez, A. G. Loukianov, and R. G. Harley, "Particle swarm optimization for discretetime inverse optimal control of a doubly fed induction generator," IEEE Transactions on Cybernetics, vol. 43, no. 6, pp. 1698-1709, 2013.

[38] Q. Niu, Z. Zhou, H. Y. Zhang, and J. Deng, “An improved quantum-behaved particle swarm optimization method for economic dispatch problems with multiple fuel options and valvepoints effects," Energies, vol. 5, no. 9, pp. 3655-3673, 2012.

[39] J. Sun, W. Fang, X. Wu, V. Palade, and W. Xu, "Quantumbehaved particle swarm optimization: analysis of individual particle behavior and parameter selection," Evolutionary Computation, vol. 20, no. 3, pp. 349-393, 2012.

[40] C. L. Chiang, "Quantum-behaved particle swarm optimization for power economic dispatch problem of units with multiple fuel option," European Journal of Engineering Research and Science, vol. 2, no. 12, 2017.

[41] X. S. Yang, Nature-Inspired Algorithms and Applied Optimization, Springer, New York, 2018.

[42] M. Premkumar and R. Sumithira, "Humpback whale assisted hybrid maximum power point tracking algorithm for partially shaded solar photovoltaic systems," Journal of Power Electronics, vol. 18, no. 6, pp. 1805-1818, 2018.

[43] M. Premkumar and R. Sowmya, "An effective maximum power point tracker for partially shaded solar photovoltaic systems," Energy Reports, vol. 5, pp. 1445-1462, 2019.

[44] M. Premkumar, A. M. Ibrahim, R. M. Kumar, and R. Sowmya, "Analysis and simulation of bio-inspired intelligent salp swarm MPPT method for the PV systems under partial shaded conditions," International Journal of Computing and Digital Systems, vol. 8, no. 5, pp. 489-496, 2020.

[45] M. H. Parvaneh and P. G. Khorasani, "A new hybrid method based on fuzzy logic for maximum power point tracking of photovoltaic systems," Energy Reports, vol. 6, pp. 1619-1632, 2020.

[46] J. Falin, "Designing DC/DC converters based on ZETA topology," Analog Applications Journal, Texas Instruments 
Incorporated, pp. 16-21, 2010, https://www.ti.com/lit/an/ slyt372/slyt372.pdf?ts=1605727121467\&ref_url=https\% 253A\%252F\%252Fwww.google.com\%252F.

[47] T. M. Leandro, R. Matthias, H. F. Marcelo, S. M. Lucas, E. B. Fábio, and K. Dirk, "Modeling and design of a linear-assisted Zeta converter," in Proc. Int. Conf. 21st European Conference on Power Electronics and Applications (EPE '19 ECCE Europe), pp. 1-10, Genova, Italy, Italy, 2019.

[48] P. Amit and T. Harpal, "Implementation of INC-PIMPPT and its comparison with INC MPPT by direct duty cycle control for solar photovoltaics employing Zeta converter," in Proc. Int. Conf. Information, Communication, Instrumentation and Control (ICICIC), pp. 1-6, Indore, India, 2017.

[49] P. R. Babu, S. R. Prasath, and R. Kiruthika, "Simulation and performance analysis of CCM Zeta converter with PID controller," in Proc. Int. Conf. Circuits, Power and Computing Technologies (ICCPCT-2015), pp. 1-7, Nagercoil, India, 2015.

[50] A. A. Ahmad, A. Abrishamifar, and M. Farzi, "A new design procedure for output LC filter of single phase inverters," in Proc. Int. Conf. Power Electronics and Intelligent Transportation System, pp. 86-91, Shenzhen, China, 2010.

[51] P. A. Dahono, A. Purwadi, and Qamaruzzaman, "An LC filter design method for single-phase PWM inverters," in Proc. Int. Conf. Power Electronics and Drive Systems, pp. 571-576, Singapore, 1995.

[52] H. S. Kim and S. K. Sul, "A novel filter design for output LC filters of PWM inverters," Journal of Power Electronics, vol. 11, no. 1, pp. 74-81, 2011.

[53] T. Aleksei, P. Eduard, and B. Juri, "Closed-loop identification of fractional-order models using FOMCON toolbox for MATLAB," in Proc. Int. Conf. 14th Biennial Baltic Electronic Conference (BEC), pp. 213-216, Tallinn, Estonia, 2014.

[54] M. Deep, K. K. Palash, and G. Apurba, "PID controller design for an interacting tank level process with time delay using MATLAB FOMCON toolbox," in Proc. Int. Conf. 2nd Control, Instrumentation, Energy \& Communication (CIEC), pp. 1-5, Kolkata, India, 2016.

[55] T. Aleksei, P. Eduard, B. Juri, and F. Jevgeni, "Fractional-order controller design and digital implementation using FOMCON toolbox for MATLAB," in Proc. IEEE Int. Conf. Computer Aided Control System Design (CACSD), pp. 340-345, Hyderabad, India, 2013.

[56] T. Aleksei, P. Eduard, and B. Juri, “A flexible MATLAB tool for optimal fractional-order PID controller design subject to specifications," in Proc. Int. Conf. 31st Chinese Control, pp. 4698-4703, Hefei, China, 2012.

[57] M. Kuhn, "Building predictive models inRUsing thecaretPackage," Journal of Statistical Software, vol. 28, no. 5, 2008.

[58] J. Zhou, E. Li, H. Wei, C. Li, Q. Qiao, and D. J. Armaghani, "Random forests and cubist algorithms for predicting shear strengths of rockfill materials," Applied Sciences, vol. 9, no. 8, p. 1621, 2019.

[59] A. E. Maxwell, T. A. Warner, and F. Fang, "Implementation of machine-learning classification in remote sensing: an applied review," International Journal of Remote Sensing, vol. 39, no. 9, pp. 2784-2817, 2018.

[60] J. K. Liu, Radial Basis Function (RBF) Neural Network Control for Mechanical Systems: Design, Analysis and MATLAB Simulation, Springer-Verlag, Berlin, 2013.

[61] B. Liang, Y. Zhu, Y. Li, P. He, and W. Li, "Adaptive nonsingular fast terminal sliding mode control for braking systems with electro-mechanical actuators based on radial basis function," Energies, vol. 10, no. 10, p. 1637, 2017.

[62] J. Zhu, Z. Cao, T. Zhang, Y. Yang, and Y. Yi, "Sufficient condition for the existence of the compact set in the RBF neural network control," IEEE Transactions on Neural Networks and Learning Systems, pp. 1-6, 2017.

[63] Z. Xu, G. Han, H. Zhu, L. Liu, and M. Guizani, “Adaptive DE algorithm for novel energy control framework based on edge computing in IIoT applications," IEEE Transactions on Industrial Informatics, pp. 1-11, 2020.

[64] Z. Huang, Z. Lin, Z. Zhu, and J. Chen, "An improved simulated annealing algorithm with excessive length penalty for fixedoutline floorplanning," IEEE Access, vol. 8, pp. 50911-50920, 2020.

[65] B. T. M. Anh, "Enhanced genetic algorithm for automatic generation of unit and integration test suite," in Proc. Int. Conf. Computing and Communication Technologies (RIVF), pp. 16, Ho Chi Minh, Vietnam, 2020.

[66] T. Djemai, P. Stolf, T. Monteil, and J. M. Pierson, “A discrete particle swarm optimization approach for energy-efficient IoT services placement over fog infrastructures," in Proc. Int. Symp. Parallel and Distributed Computing (ISPDC), pp. 32 40, Amsterdam, Netherlands, 2019. 